Kragujevac Journal of Mathematics

Volume 40(2) (2016), Pages 172-191.

\title{
FRACTIONAL HERMITE-HADAMARD TYPE INEQUALITIES FOR FUNCTIONS WHOSE SECOND DERIVATIVE ARE $(s, r)$-CONVEX IN THE SECOND SENSE
}

\author{
K. BOUKERRIOUA ${ }^{1}$, T. CHIHEB ${ }^{1}$, AND B. MEFTAH ${ }^{2}$
}

\begin{abstract}
In this paper the authors introduce a new class of convex functions called $(s, r)$-convex functions in the second sense and establish some new HermiteHadamard type inequalities involving Riemann-Liouville integral operator.
\end{abstract}

\section{INTRODUCTION}

One of the most well-known inequalities in mathematics for convex functions is so called Hermite-Hadamard integral inequality

$$
f\left(\frac{a+b}{2}\right) \leq \frac{1}{b-a} \int_{a}^{b} f(x) d x \leq \frac{f(a)+f(b)}{2}
$$

where $f$ is a real continuous convex function on the finite interval $[a, b]$. If the function $f$ is concave, then (1.1) holds in the reverse direction (see [24]).

The Hermite-Hadamard inequality play an important role in nonlinear analysis and optimization. The above double inequality has attracted many researchers, various generalizations, refinements, extensions and variants of (1.1) have appeared in the literature, via classical integration and fractional calculus, we can mention the works [2-4,6-12,14-17, 19-23,25-32] and the references cited therein.

Recently, Wang et al. [29], proved the following Hermite-Hadamard's inequalities whose power of second derivatives are $r$-convex via fractional integrals.

Key words and phrases. Hermite-Hadamard inequality, convex functions, Riemann-Liouville integral operator.

2010 Mathematics Subject Classification. Primary 26D15, Secondary 26D20, 39A12.

Received: November 9, 2015.

Accepted: May 20, 2016. 
Theorem 1.1 ([29], Theorem 4.1). Let $f:\left[0, b^{*}\right] \rightarrow \mathbb{R}$ be a twice differentiable mapping with $b^{*}>0$. If $\left|f^{\prime \prime}\right|^{q}(q>1)$ is measurable and $r$-convex on $[a, b]$, for some fixed $0 \leq r<\infty$ and $0 \leq a<b$, then the following inequality holds for fractional integrals:

$$
\left|\frac{f(a)+f(b)}{2}-\frac{\Gamma(\alpha+1)}{2(b-a)^{\alpha}}\left[J_{a^{+}}^{\alpha} f(b)+J_{b^{-}}^{\alpha} f(a)\right]\right| \leq I_{r}
$$

where

$$
I_{r}=\frac{2^{\frac{1-r-q r}{q r}}(b-a)^{2}}{\alpha+1}\left(1-\frac{2}{p \alpha+p+1}\right)^{\frac{1}{p}}\left(\left|f^{\prime \prime}(a)\right|^{q}+\left|f^{\prime \prime}(b)\right|^{q}\right)^{\frac{1}{q}}\left(\frac{r}{r+1}\right)^{\frac{1}{q}}
$$

for $0<r \leq 1$, and

$$
I_{r}=\frac{(b-a)^{2}}{2(\alpha+1)}\left(1-\frac{2}{p \alpha+p+1}\right)^{\frac{1}{p}}\left(\left|f^{\prime \prime}(a)\right|^{q}+\left|f^{\prime \prime}(b)\right|^{q}\right)^{\frac{1}{q}}\left(\frac{r}{r+1}\right)^{\frac{1}{q}}
$$

for $r>1$;

$$
I_{0}=\frac{(b-a)^{2}}{2(\alpha+1)}\left(1-\frac{2}{p \alpha+p+1}\right)^{\frac{1}{p}}\left(\frac{\left|f^{\prime \prime}(a)\right|^{q}-\left|f^{\prime \prime}(b)\right|^{q}}{q \ln \left|f^{\prime \prime}(a)\right|-q \ln \left|f^{\prime \prime}(b)\right|}\right)^{\frac{1}{q}}
$$

for $\left|f^{\prime \prime}(a)\right| \neq\left|f^{\prime \prime}(b)\right|$, and

$$
I_{0}=\frac{(b-a)^{2}}{2(\alpha+1)}\left|f^{\prime \prime}(a)\right|\left(1-\frac{2}{p \alpha+p+1}\right)^{\frac{1}{p}}
$$

for $\left|f^{\prime \prime}(a)\right|=\left|f^{\prime \prime}(b)\right|$; and $\frac{1}{p}+\frac{1}{q}=1$.

Theorem 1.2 ([29], Theorem 4.2). Let $f:\left[0, b^{*}\right] \rightarrow \mathbb{R}$ be a twice differentiable mapping with $b^{*}>0$. If $\left|f^{\prime \prime}\right|^{q}(q>1)$ is measurable and $r$-convex on $[a, b]$, for some fixed $0 \leq r<\infty$ and $0 \leq a<b$, then the following inequality holds for fractional integrals:

where

$$
\left|\frac{f(a)+f(b)}{2}-\frac{\Gamma(\alpha+1)}{2(b-a)^{\alpha}}\left[J_{a^{+}}^{\alpha} f(b)+J_{b^{-}}^{\alpha} f(a)\right]\right| \leq I_{r}
$$

$$
\begin{aligned}
I_{r}= & \frac{2^{\frac{1-r-q r}{q r}}(b-a)^{2}}{\alpha+1}\left(\left|f^{\prime \prime}(a)\right|^{q}+\left|f^{\prime \prime}(b)\right|^{q}\right)^{\frac{1}{q}}\left[\frac{r}{r+1}-\beta\left(\frac{r}{r+1}, \alpha q+q+1\right)\right. \\
& \left.-\frac{r}{\alpha q r+q r+r+1+1}\right]^{\frac{1}{q}},
\end{aligned}
$$

for $0<r \leq 1$, and

$$
\begin{aligned}
I_{r}= & \frac{(b-a)^{2}}{2(\alpha+1)}\left(\left|f^{\prime \prime}(a)\right|^{q}+\left|f^{\prime \prime}(b)\right|^{q}\right)^{\frac{1}{q}}\left[\frac{r}{r+1}-\beta\left(\frac{r}{r+1}, \alpha q+q+1\right)\right. \\
& \left.-\frac{r}{\alpha q r+q r+r+1+1}\right]^{\frac{1}{q}},
\end{aligned}
$$


for $r>1$;

$$
\begin{aligned}
I_{0}= & \frac{(b-a)^{2}}{2(\alpha+1)}\left[\frac{\left|f^{\prime \prime}(a)\right|^{q}-\left|f^{\prime \prime}(b)\right|^{q}}{q\left(\ln \left|f^{\prime \prime}(a)\right|-\ln \left|f^{\prime \prime}(b)\right|\right)}\right. \\
& \left.-\sum_{i=1}^{\infty} \frac{\left(\ln \left|f^{\prime \prime}(a)\right|-\ln \left|f^{\prime \prime}(b)\right|\right)^{i-1}}{(q \alpha+q+1)_{i}}\left[q^{i-1}\left|f^{\prime \prime}(b)\right|^{q}-(-q)^{i-1}\left|f^{\prime \prime}(a)\right|^{q}\right]\right]^{\frac{1}{q}},
\end{aligned}
$$

for $\left|f^{\prime \prime}(a)\right| \neq\left|f^{\prime \prime}(b)\right|$, and

$$
I_{0}=\frac{(b-a)^{2}}{2(\alpha+1)}\left|f^{\prime \prime}(a)\right|\left(1-\frac{2}{q \alpha+q+1}\right)^{\frac{1}{q}}
$$

for $\left|f^{\prime \prime}(a)\right|=\left|f^{\prime \prime}(b)\right|$; and $\frac{1}{p}+\frac{1}{q}=1$.

In [18], Lin et al. proved the following Hermite-Hadamard's inequalities whose second derivatives are $r$-convex via fractional integrals.

Theorem 1.3 ([18], Proposition 4.2). Let $f:[a, b] \rightarrow \mathbb{R}$ be a twice differentiable mapping with $0 \leq a<b$. If $\left|f^{\prime \prime}\right|$ is integrable and $r$-convex on $[a, b]$ for some fixed $0 \leq r<\infty$, then the following inequality for fractional integrals holds

$$
\left|\frac{\Gamma(\alpha+1)}{2(b-a)^{\alpha}}\left[J_{a^{+}}^{\alpha} f(b)+J_{b^{-}}^{\alpha} f(a)\right]-\frac{f(a)+f(b)}{2}\right| \leq K_{r},
$$

where

$$
K_{r}=2^{\frac{1}{r}-2}(b-a)^{2} \frac{\left|f^{\prime \prime}(a)\right|+\left|f^{\prime \prime}(b)\right|}{\alpha+1}\left[\frac{r}{r+1}-\frac{r}{\alpha r+2 r+1}-\beta\left(\frac{1}{r}+1, \alpha+2\right)\right],
$$

for $0<r \leq 1$,

$$
K_{r}=(b-a)^{2} \frac{\left|f^{\prime \prime}(a)\right|+\left|f^{\prime \prime}(b)\right|}{\alpha+1}\left[\frac{r}{r+1}-\frac{r}{\alpha r+2 r+1}-\beta\left(\frac{1}{r}+1, \alpha+2\right)\right],
$$

for $r>1$,

$$
K_{r}=\frac{(b-a)^{2}\left|f^{\prime \prime}(b)\right|}{2(\alpha+1)}\left[\frac{|k|-1}{\ln |k|}-|k| \sum_{i=1}^{\infty}(-1)^{i-1} \frac{(\ln |k|)^{i-1}}{(\alpha+1)_{i}}-\sum_{i=1}^{\infty} \frac{(\ln |k|)^{i-1}}{(\alpha+1)_{i}}\right],
$$

for $r=0$, and $k=\left|f^{\prime \prime}(a)\right| /\left|f^{\prime \prime}(b)\right|$.

Theorem 1.4 ([18], Proposition 4.5). Let $f:[a, b] \rightarrow \mathbb{R}$ be a twice differentiable mapping with $0 \leq a<b$. If $\left|f^{\prime \prime}\right|^{q}, q>1$ is integrable and $r$-convex on $[a, b]$ for some fixed $0 \leq r<\infty$, then the following inequality for fractional integrals holds

$$
\left|\frac{\Gamma(\alpha+1)}{2(b-a)^{\alpha}}\left[J_{a^{+}}^{\alpha} f(b)+J_{b^{-}}^{\alpha} f(a)\right]-\frac{f(a)+f(b)}{2}\right| \leq K_{r},
$$


where

$$
K_{r}=\frac{2^{\frac{1}{q r}-1}(b-a)^{2}}{\alpha+1}\left[1-\frac{2(q-1)}{q \alpha+2 q-1}\right]^{1-\frac{1}{q}}\left[\left(\left|f^{\prime \prime}(a)\right|^{q}+\left|f^{\prime \prime}(b)\right|^{q}\right) \frac{r}{r+1}\right]^{\frac{1}{q}},
$$

for $0<r \leq 1$,

$$
K_{r}=\frac{2^{\frac{1}{q}-1}(b-a)^{2}}{\alpha+1}\left[1-\frac{2(q-1)}{q \alpha+2 q-1}\right]^{1-\frac{1}{q}}\left[\left(\left|f^{\prime \prime}(a)\right|^{q}+\left|f^{\prime \prime}(b)\right|^{q}\right) \frac{r}{r+1}\right]^{\frac{1}{q}},
$$

for $r>1$,

$$
K_{r}=\frac{2^{\frac{1}{q}-1}(b-a)^{2}\left|f^{\prime \prime}(b)\right|}{\alpha+1}\left[1-\frac{2(q-1)}{q \alpha+2 q-1}\right]^{1-\frac{1}{q}} \quad\left(\frac{|k|^{q}-1}{q \ln |k|}\right)^{\frac{1}{q}},
$$

for $r=0$, and $k=\left|f^{\prime \prime}(a)\right| /\left|f^{\prime \prime}(b)\right|$.

Motivated by the above results, in this paper we extend those results for a new class of convexity called $(s, r)$-convex functions in the second sense.

\section{Preliminaries}

In this section we recall some concepts of convexity which are well known in the literature. Let $I$ be an interval of $\mathbb{R}$.

Definition 2.1. [24] A function $f: I \rightarrow \mathbb{R}$ is said to be convex, if

$$
f(t x+(1-t) y) \leq t f(x)+(1-t) f(y),
$$

holds for all $x, y \in I$ and all $t \in[0,1]$.

Definition 2.2. [24] A positive function $f: I \rightarrow \mathbb{R}$ is said to be logarithmically convex, if

$$
f(t x+(1-t) y) \leq[f(x)]^{t}[f(y)]^{(1-t)},
$$

holds for all $x, y \in I$ and all $t \in[0,1]$.

Definition 2.3. [5] A nonnegative function $f: I \subseteq[0, \infty) \rightarrow \mathbb{R}$ is said to be $s$-convex in the second sense on $I$, for some fixed $s \in(0,1]$, if

$$
f(t x+(1-t) y) \leq t^{s} f(x)+(1-t)^{s} f(y),
$$

holds for all $x, y \in I$ and $t \in[0,1]$.

Definition 2.4. [1] A positive function $f: I \subseteq[0, \infty) \rightarrow \mathbb{R}$ is said to be $s$ logarithmically convex function in the second sense on $I$ for some fixed $s \in(0,1]$, if

$$
f(t x+(1-t) y) \leq[f(x)]^{t^{s}}[f(y)]^{(1-t)^{s}},
$$

holds for all $x, y \in I$ and $t \in[0,1]$. 
Definition 2.5. [23] A positive function $f: I \subseteq[0, \infty) \rightarrow \mathbb{R}$ is said to be $r$-convex on $I$, if

$$
f(t x+(1-t) y) \leq \begin{cases}{\left[t f^{r}(x)+(1-t) f^{r}(y)\right]^{\frac{1}{r}},} & \text { if } r \neq 0 \\ {[f(x)]^{1-t}[f(y)]^{t \lambda},} & \text { if } r=0\end{cases}
$$

holds for all $x, y \in I$ and $t \in[0,1]$.

Definition 2.6. [13,14] Let $f \in L_{1}[a, b]$. The Riemann-Liouville integrals $J_{a^{+}}^{\alpha} f$ and $J_{b^{-}}^{\alpha} f$ of order $\alpha>0$ with $a \geq 0$ are defined by

$$
\begin{aligned}
& J_{a^{+}}^{\alpha} f(x)=\frac{1}{\Gamma(\alpha)} \int_{a}^{x}(x-t)^{\alpha-1} f(t) d t, \quad x>a, \\
& J_{b^{-}}^{\alpha} f(x)=\frac{1}{\Gamma(\alpha)} \int_{x}^{b}(t-x)^{\alpha-1} f(t) d t, \quad b>x,
\end{aligned}
$$

respectively, where $\Gamma(\alpha)=\int_{0}^{\infty} e^{-t} t^{\alpha-1} d t$, is the Gamma function and $J_{a^{+}}^{0} f(x)=$ $J_{b^{-}}^{0} f(x)=f(x)$.

Lemma 2.1. [29] For $\alpha>0$ and $k>0, z>0$, we have

$$
\begin{aligned}
J(\alpha, k) & =\int_{0}^{1}(1-t)^{\alpha-1} k^{t} d t=\sum_{i=1}^{\infty} \frac{(\ln k)^{i-1}}{(\alpha)_{i}}<\infty, \\
H(\alpha, k, z) & =\int_{0}^{1} t^{\alpha-1} k^{t} d t=z^{\alpha} k^{z} \sum_{i=1}^{\infty} \frac{(-z \ln k)^{i-1}}{(\alpha)_{i}}<\infty,
\end{aligned}
$$

where $(\alpha)_{i}=\prod_{j=0}^{i-1}(\alpha+j)$.

Lemma 2.2. [29] Let $f:[a, b] \rightarrow \mathbb{R}$ be a twice differentiable mapping on $(a, b)$ with $0 \leq a<b$. If $f \in L[a, b]$, then the following equality for fractional integrals holds:

$$
\begin{aligned}
& \frac{f(a)+f(b)}{2}-\frac{\Gamma(\alpha+1)}{2(b-a)^{\alpha}}\left[J_{a^{+}}^{\alpha} f(b)+J_{b^{-}}^{\alpha} f(a)\right] \\
= & \frac{(b-a)^{2}}{2(\alpha+1)} \int_{0}^{1}\left[1-(1-t)^{\alpha+1}-t^{\alpha+1}\right] f^{\prime \prime}(t a+(1-t) b) d t .
\end{aligned}
$$

Also, we recall that the Euler Beta function is defined as follows

$$
\beta(x, y)=\int_{0}^{1} t^{x-1}(1-t)^{y-1} d t=\frac{\Gamma(x) \Gamma(y)}{\Gamma(x+y)} .
$$




\section{MAin Results}

In order to prove our results, we first introduce a new concept of convexity called $(s, r)$-convexity in the second sense.

Definition 3.1. A positive function $f: I \subseteq[0, \infty) \rightarrow \mathbb{R}$, is said to be $(s, r)$-convex in the second sense, if

$$
f(t x+(1-t) y) \leq \begin{cases}{\left[t^{s} f^{r}(x)+(1-t)^{s} f^{r}(y)\right]^{\frac{1}{r}},} & \text { if } r \neq 0 \\ {[f(x)]^{t^{s}}[f(y)]^{(1-t)^{s}},} & \text { if } r=0\end{cases}
$$

holds, for some fixed $s \in(0,1], t \in[0,1]$ and all $x, y \in I$.

Example 3.1. We define the function $g$ as follows:

$$
g(t)= \begin{cases}a^{\frac{1}{r}}, & \text { if } t=0 \\ \left(b t^{s}+c\right)^{\frac{1}{r}}, & \text { if } t>0\end{cases}
$$

where $a, b, c, r \in \mathbb{R}$ and $s \in(0,1]$ such that $b \geq 0$ and $0 \leq c \leq a$ and $r>0$.

The function $g$ is $(s, r)$-convex in the second sense, because

$$
g^{r}(t)=f(t)= \begin{cases}a, & \text { if } t=0 \\ b t^{s}+c, & \text { if } t>0\end{cases}
$$

is $s$-convex in the second sense, for more details see [12].

Remark 3.1. Obviously Definition 3.1, recapture all definitions cited above for wellchosen values of $s$ and $r$.

Theorem 3.1. Let $f:[a, b] \rightarrow(0, \infty)$ be twice differentiable mapping with $a<b$, $f^{\prime \prime} \in L([a, b])$. If $\left|f^{\prime \prime}\right|$ is $(s, r)$-convex function in the second sense for some fixed $s \in(0,1]$, then the following inequality for fractional integrals with $\alpha>0$

$$
\left|\frac{f(a)+f(b)}{2}-\frac{\Gamma(\alpha+1)}{2(b-a)^{\alpha}}\left[J_{a^{+}}^{\alpha} f(b)+J_{b^{-}}^{\alpha} f(a)\right]\right| \leq T,
$$

where

$$
\begin{aligned}
T & =\frac{c(r)(b-a)^{2}}{2(\alpha+1)}\left[\frac{r^{2}(\alpha+1)}{(s+r)(s+r(\alpha+2))}-\beta\left(\frac{s}{r}+1, \alpha+2\right)\right]\left[\left|f^{\prime \prime}(a)\right|+\left|f^{\prime \prime}(b)\right|\right], \\
\text { for } r> & 0, \\
T & =\frac{(b-a)^{2}}{2(\alpha+1)} E(a, b, s)\left[\frac{1}{s\left|f^{\prime \prime}(b)\right|} \times \frac{\left|f^{\prime \prime}(a)\right|^{s}-\left|f^{\prime \prime}(b)\right|^{s}}{\ln \left|f^{\prime \prime}(a)\right|-\ln \left|f^{\prime \prime}(b)\right|}-\sum_{i=1}^{\infty} \frac{\left(\ln \left|\frac{f^{\prime \prime}(a)}{f^{\prime \prime}(b)}\right|^{s}\right)^{i-1}}{(\alpha+2)_{i}}\right. \\
& \left.-\left|\frac{f^{\prime \prime}(a)}{f^{\prime \prime}(b)}\right|^{s} \sum_{i=1}^{\infty}(-1)^{i-1} \frac{\left(\ln \left|\frac{f^{\prime \prime}(a)}{f^{\prime \prime}(b)}\right|^{s}\right)^{i-1}}{(\alpha+2)_{i}}\right],
\end{aligned}
$$


for $r=0$ and $\left|f^{\prime \prime}(a)\right| \neq\left|f^{\prime \prime}(b)\right|$, and

$$
T=\frac{\alpha(b-a)^{2}}{2(\alpha+1)(\alpha+2)} N(b, s)
$$

for $r=0$ and $\left|f^{\prime \prime}(a)\right|=\left|f^{\prime \prime}(b)\right|$, holds for $r \geq 0$ where

$$
\begin{gathered}
c(r)= \begin{cases}1, & \text { if } r \geq 1, \\
2^{\frac{1}{r}-1}, & \text { if } 0<r \leq 1,\end{cases} \\
E(a, b, s)= \begin{cases}\left|f^{\prime \prime}(b)\right|^{s}, & \text { if }\left|f^{\prime \prime}(a)\right|,\left|f^{\prime \prime}(b)\right| \leq 1, \\
\left|f^{\prime \prime}(b)\right|, & \text { if }\left|f^{\prime \prime}(a)\right| \leq 1 \leq\left|f^{\prime \prime}(b)\right|, \\
\left|f^{\prime \prime}(a)\right|^{1-s}\left|f^{\prime \prime}(b)\right|^{s}, & \text { if }\left|f^{\prime \prime}(b)\right| \leq 1 \leq\left|f^{\prime \prime}(a)\right|, \\
\left|f^{\prime \prime}(a)\right|^{1-s}\left|f^{\prime \prime}(b)\right|, & \text { if }\left|f^{\prime \prime}(a)\right|,\left|f^{\prime \prime}(b)\right| \geq 1,\end{cases}
\end{gathered}
$$

$$
N(b, s)= \begin{cases}\left|f^{\prime \prime}(b)\right|^{s}, & \text { if }\left|f^{\prime \prime}(a)\right|=\left|f^{\prime \prime}(b)\right| \leq 1 \\ \left|f^{\prime \prime}(b)\right|^{2-s}, & \text { if }\left|f^{\prime \prime}(a)\right|=\left|f^{\prime \prime}(b)\right| \geq 1\end{cases}
$$

and $(\alpha+2)_{i}=\prod_{j=1}^{i-1}(\alpha+2+j)$.

Proof. From Lemma 2.2 and property of the modulus, we have

$$
\begin{aligned}
& \left|\frac{f(a)+f(b)}{2}-\frac{\Gamma(\alpha+1)}{2(b-a)^{\alpha}}\left[J_{a^{+}}^{\alpha} f(b)+J_{b^{-}}^{\alpha} f(a)\right]\right| \\
\leq & \frac{(b-a)^{2}}{2(\alpha+1)} \int_{0}^{1}\left[1-(1-t)^{\alpha+1}-t^{\alpha+1}\right]\left|f^{\prime \prime}(t a+(1-t) b)\right| d t .
\end{aligned}
$$

Case 1: $r>0$.

Since $\left|f^{\prime \prime}\right|$ is $(s, r)$-convex in the second sense, we get

$$
\left|f^{\prime \prime}(t a+(1-t) b)\right| \leq\left[t^{s}\left|f^{\prime \prime}(a)\right|^{r}+(1-t)^{s}\left|f^{\prime \prime}(b)\right|^{r}\right]^{\frac{1}{r}},
$$

it is easy to see that

$$
\left|f^{\prime \prime}(t a+(1-t) b)\right| \leq c(r)\left[t^{\frac{s}{r}}\left|f^{\prime \prime}(a)\right|+(1-t)^{\frac{s}{r}}\left|f^{\prime \prime}(b)\right|\right],
$$

where $c(r)$ is defined as in (3.2).

Substituting (3.7) into (3.5), we obtain

$$
\begin{aligned}
& \frac{(b-a)^{2}}{2(\alpha+1)} \int_{0}^{1}\left[1-(1-t)^{\alpha+1}-t^{\alpha+1}\right]\left|f^{\prime \prime}(t a+(1-t) b)\right| d t \\
\leq & \frac{c(r)(b-a)^{2}}{2(\alpha+1)}\left|f^{\prime \prime}(a)\right| \int_{0}^{1}\left[t^{\frac{s}{r}}-t^{\frac{s}{r}}(1-t)^{\alpha+1}-t^{\frac{s}{r}+\alpha+1}\right] d t
\end{aligned}
$$




$$
\begin{aligned}
& \quad+\frac{c(r)(b-a)^{2}}{2(\alpha+1)}\left|f^{\prime \prime}(b)\right| \int_{0}^{1}\left[(1-t)^{\frac{s}{r}}-(1-t)^{\frac{s}{r}+\alpha+1}-t^{\alpha+1}(1-t)^{\frac{s}{r}}\right] d t \\
& =\frac{c(r)(b-a)^{2}}{2(\alpha+1)}\left|f^{\prime \prime}(a)\right|\left[\frac{r^{2}(\alpha+1)}{(s+r)(s+r(\alpha+2))}-\beta\left(\frac{s}{r}+1, \alpha+2\right)\right] \\
& \quad+\frac{c(r)(b-a)^{2}}{2(\alpha+1)}\left|f^{\prime \prime}(b)\right|\left[\frac{r^{2}(\alpha+1)}{(s+r)(s+r(\alpha+2))}-\beta\left(\alpha+2, \frac{s}{r}+1\right)\right] \\
& =\frac{c(r)(b-a)^{2}}{2(\alpha+1)}\left[\frac{r^{2}(\alpha+1)}{(s+r)(s+r(\alpha+2))}-\beta\left(\frac{s}{r}+1, \alpha+2\right)\right] \\
& \quad \times\left[\left|f^{\prime \prime}(a)\right|+\left|f^{\prime \prime}(b)\right|\right] .
\end{aligned}
$$

Case 2: $r=0$.

Since $\left|f^{\prime \prime}\right|$ is $(s, 0)$-convex, we have

$$
\left|f^{\prime \prime}(t a+(1-t) b)\right| \leq\left|f^{\prime \prime}(a)\right|^{t^{s}}\left|f^{\prime \prime}(b)\right|^{(1-t)^{s}},
$$

using the fact that $\phi^{t^{s}} \leq \phi^{s t}$ and $\psi^{t^{s}} \leq \psi^{s t+1-s}$ is valid for all $0<\phi \leq 1 \leq \psi$ and $t, s \in(0,1]$, we get

$$
\left|f^{\prime \prime}(t a+(1-t) b)\right| \leq E(a, b, s)\left(\frac{\left|f^{\prime \prime}(a)\right|}{\left|f^{\prime \prime}(b)\right|}\right)^{s t}, \quad \text { if }\left|f^{\prime \prime}(a)\right| \neq\left|f^{\prime \prime}(b)\right|,
$$

and

$$
\left|f^{\prime \prime}(t a+(1-t) b)\right| \leq N(b, s), \quad \text { if }\left|f^{\prime \prime}(a)\right|=\left|f^{\prime \prime}(b)\right|
$$

where $E(a, b, s)$ and $N(b, s)$ are defined as in (3.3) and (3.4) respectively.

Substituting (3.10) into (3.5), we obtain

$$
\begin{aligned}
& \left|\frac{f(a)+f(b)}{2}-\frac{\Gamma(\alpha+1)}{2(b-a)^{\alpha}}\left[J_{a^{+}}^{\alpha} f(b)+J_{b^{-}}^{\alpha} f(a)\right]\right| \\
\leq & \frac{(b-a)^{2} E(a, b, s)}{2(\alpha+1)} \int_{0}^{1}\left[1-(1-t)^{\alpha+1}-t^{\alpha+1}\right]\left(\frac{\left|f^{\prime \prime}(a)\right|}{\left|f^{\prime \prime}(b)\right|}\right)^{s t} d t \\
= & \frac{(b-a)^{2} E(a, b, s)}{2(\alpha+1)}\left[\int_{0}^{1}\left(\frac{\left|f^{\prime \prime}(a)\right|}{\left|f^{\prime \prime}(b)\right|}\right)^{s t} d t\right. \\
& \left.-\int_{0}^{1}(1-t)^{\alpha+1}\left(\frac{\left|f^{\prime \prime}(a)\right|}{\left|f^{\prime \prime}(b)\right|}\right)^{s t} d t-\int_{0}^{1} t^{\alpha+1}\left(\frac{\left|f^{\prime \prime}(a)\right|}{\left|f^{\prime \prime}(b)\right|}\right)^{s t} d t\right] .
\end{aligned}
$$


From Lemma 2.1, with $z=1$, we get

$$
\begin{aligned}
& \int_{0}^{1}(1-t)^{\alpha+1}\left|\left(\frac{f^{\prime \prime}(a)}{f^{\prime \prime}(b)}\right)^{s}\right|^{t} d t=\sum_{i=1}^{\infty} \frac{\left(\ln \left|\left(\frac{f^{\prime \prime}(a)}{f^{\prime \prime}(b)}\right)^{s}\right|\right)^{i-1}}{(\alpha+2)_{i}}, \\
& \int_{0}^{1} t^{\alpha+1}\left|\left(\frac{f^{\prime \prime}(a)}{f^{\prime \prime}(b)}\right)^{s}\right|^{t} d t=\left|\frac{f^{\prime \prime}(a)}{f^{\prime \prime}(b)}\right|^{s} \sum_{i=1}^{\infty}(-1)^{i-1} \frac{\left(\ln \left|\frac{f^{\prime \prime}(a)}{f^{\prime \prime}(b)}\right|^{s}\right)^{i-1}}{(\alpha+2)_{i}},
\end{aligned}
$$

and

$$
\begin{aligned}
\int_{0}^{1}\left|\left(\frac{f^{\prime \prime}(a)}{f^{\prime \prime}(b)}\right)^{s}\right|^{t} d t & =\frac{\left|\frac{f^{\prime \prime}(a)}{f^{\prime \prime}(b)}\right|^{s}-1}{\ln \left|\frac{f^{\prime \prime}(a)}{f^{\prime \prime}(b)}\right|^{s}} \\
& =\frac{1}{s\left|f^{\prime \prime}(b)\right|} \times \frac{\left|f^{\prime \prime}(a)\right|^{s}-\left|f^{\prime \prime}(b)\right|^{s}}{\ln \left|f^{\prime \prime}(a)\right|-\ln \left|f^{\prime \prime}(b)\right|}
\end{aligned}
$$

using (3.13), (3.14) and (3.15) in (3.12), we obtain

$$
\begin{aligned}
& \left|\frac{f(a)+f(b)}{2}-\frac{\Gamma(\alpha+1)}{2(b-a)^{\alpha}}\left[J_{a^{+}}^{\alpha} f(b)+J_{b^{-}}^{\alpha} f(a)\right]\right| \\
& \leq \frac{(b-a)^{2}}{2(\alpha+1)} E(a, b, s)\left[\frac{1}{s\left|f^{\prime \prime}(b)\right|} \times \frac{\left|f^{\prime \prime}(a)\right|^{s}-\left|f^{\prime \prime}(b)\right|^{s}}{\ln \left|f^{\prime \prime}(a)\right|-\ln \left|f^{\prime \prime}(b)\right|}\right. \\
& \left.\quad-\sum_{i=1}^{\infty} \frac{\left(\ln \left|\frac{f^{\prime \prime}(a)}{f^{\prime \prime}(b)}\right|^{s}\right)^{i-1}}{(\alpha+2)_{i}}-\left|\frac{f^{\prime \prime}(a)}{f^{\prime \prime}(b)}\right|^{s} \sum_{i=1}^{\infty}(-1)^{i-1} \frac{\left(\ln \left|\frac{f^{\prime \prime}(a)}{f^{\prime \prime}(b)}\right|^{s}\right)^{i-1}}{(\alpha+2)_{i}}\right] .
\end{aligned}
$$

Now, substituting (3.11) into (3.5), we get

$$
\left|\frac{f(a)+f(b)}{2}-\frac{\Gamma(\alpha+1)}{2(b-a)^{\alpha}}\left[J_{a^{+}}^{\alpha} f(b)+J_{b^{-}}^{\alpha} f(a)\right]\right| \leq \frac{\alpha(b-a)^{2}}{2(\alpha+1)(\alpha+2)} N(b, s) .
$$

From (3.8), (3.16) and (3.17) we obtain the desired inequality in (3.1). The proof is complete.

Remark 3.2. With the same assumptions of Theorem 3.1, if $\left|f^{\prime \prime}(x)\right| \leq M_{2}$ on $[a, b]$, inequality (3.1) becomes

$$
\left|\frac{f(a)+f(b)}{2}-\frac{\Gamma(\alpha+1)}{2(b-a)^{\alpha}}\left[J_{a^{+}}^{\alpha} f(b)+J_{b^{-}}^{\alpha} f(a)\right]\right| \leq T
$$

where

$$
T=\frac{c(r)(b-a)^{2}}{(\alpha+1)}\left[\frac{r^{2}(\alpha+1)}{(s+r)(s+r(\alpha+2))}-\beta\left(\frac{s}{r}+1, \alpha+2\right)\right] M_{2},
$$


for $r>0$, and

for $r=0$.

$$
T=\frac{\alpha(b-a)^{2} M_{2}}{2(\alpha+1)(\alpha+2)}
$$

Corollary 3.1. Let $f:[a, b] \rightarrow(0, \infty)$ be twice differentiable mapping with $a<b$, $f^{\prime \prime} \in L([a, b])$. If $\left|f^{\prime \prime}\right|$ is convex function, then the following inequality for fractional integrals with $\alpha>0$, holds

$$
\begin{aligned}
& \left|\frac{f(a)+f(b)}{2}-\frac{\Gamma(\alpha+1)}{2(b-a)^{\alpha}}\left[J_{a^{+}}^{\alpha} f(b)+J_{b^{-}}^{\alpha} f(a)\right]\right| \\
\leq & \frac{\alpha(b-a)^{2}}{4(\alpha+1)(\alpha+2)}\left[\left|f^{\prime \prime}(a)\right|+\left|f^{\prime \prime}(b)\right|\right] .
\end{aligned}
$$

Corollary 3.2. Let $f:[a, b] \rightarrow(0, \infty)$ be twice differentiable mapping with $a<b$, $f^{\prime \prime} \in L([a, b])$. If $\left|f^{\prime \prime}\right|$ is s-convex function in the second sense for some fixed $s \in(0,1]$, then the following inequality for fractional integrals with $\alpha>0$, holds

$$
\begin{aligned}
& \left|\frac{f(a)+f(b)}{2}-\frac{\Gamma(\alpha+1)}{2(b-a)^{\alpha}}\left[J_{a^{+}}^{\alpha} f(b)+J_{b^{-}}^{\alpha} f(a)\right]\right| \\
\leq & \frac{(b-a)^{2}}{2(\alpha+1)}\left[\frac{(\alpha+1)}{(s+1)(s+\alpha+2)}-\beta(s+1, \alpha+2)\right]\left[\left|f^{\prime \prime}(a)\right|+\left|f^{\prime \prime}(b)\right|\right] .
\end{aligned}
$$

Corollary 3.3. Let $f:[a, b] \rightarrow(0, \infty)$ be twice differentiable mapping with $a<b$, $f^{\prime \prime} \in L([a, b])$. If $\left|f^{\prime \prime}\right|$ is $r$-convex function, then the following inequality for fractional integrals with $\alpha>0$, holds

$$
\left|\frac{f(a)+f(b)}{2}-\frac{\Gamma(\alpha+1)}{2(b-a)^{\alpha}}\left[J_{a^{+}}^{\alpha} f(b)+J_{b^{-}}^{\alpha} f(a)\right]\right| \leq T
$$

where

$$
T=\frac{c(r)(b-a)^{2}}{2(\alpha+1)}\left[\frac{r^{2}(\alpha+1)}{(1+r)(1+r(\alpha+2))}-\beta\left(\frac{1}{r}+1, \alpha+2\right)\right]\left[\left|f^{\prime \prime}(a)\right|+\left|f^{\prime \prime}(b)\right|\right],
$$

for $r>0$,

$$
\begin{aligned}
T= & \frac{(b-a)^{2}}{2(\alpha+1)}\left[\frac{\left|f^{\prime \prime}(a)\right|-\left|f^{\prime \prime}(b)\right|}{\ln \left|f^{\prime \prime}(a)\right|-\ln \left|f^{\prime \prime}(b)\right|}-\left|f^{\prime \prime}(b)\right| \sum_{i=1}^{\infty} \frac{\left(\ln \left|\frac{f^{\prime \prime}(a)}{f^{\prime \prime}(b)}\right|\right)^{i-1}}{(\alpha+2)_{i}}\right. \\
& \left.-\left|f^{\prime \prime}(a)\right| \sum_{i=1}^{\infty}(-1)^{i-1} \frac{\left(\ln \left|\frac{f^{\prime \prime}(a)}{f^{\prime \prime}(b)}\right|\right)^{i-1}}{(\alpha+2)_{i}}\right],
\end{aligned}
$$

for $r=0$ and $\left|f^{\prime \prime}(a)\right| \neq\left|f^{\prime \prime}(b)\right|$,

$$
T=\frac{\alpha(b-a)^{2}\left|f^{\prime \prime}(b)\right|}{2(\alpha+1)(\alpha+2)}
$$


for $r=0$ and $\left|f^{\prime \prime}(a)\right|=\left|f^{\prime \prime}(b)\right|$.

Remark 3.3. Corollary 3.3, is similar to the Proposition 4.2 from [18].

Corollary 3.4. Let $f:[a, b] \rightarrow(0, \infty)$ be twice differentiable mapping with $a<b$, $f^{\prime \prime} \in L([a, b])$. If $\left|f^{\prime \prime}\right|$ is logarithmically convex function, then the following inequality for fractional integrals with $\alpha>0$, holds

$$
\left|\frac{f(a)+f(b)}{2}-\frac{\Gamma(\alpha+1)}{2(b-a)^{\alpha}}\left[J_{a^{+}}^{\alpha} f(b)+J_{b^{-}}^{\alpha} f(a)\right]\right| \leq T,
$$

where

$$
\begin{aligned}
T= & \frac{(b-a)^{2}}{2(\alpha+1)}\left[\frac{\left|f^{\prime \prime}(a)\right|-\left|f^{\prime \prime}(b)\right|}{\ln \left|f^{\prime \prime}(a)\right|-\ln \left|f^{\prime \prime}(b)\right|}-\left|f^{\prime \prime}(b)\right| \sum_{i=1}^{\infty} \frac{\left(\ln \left|\frac{f^{\prime \prime}(a)}{f^{\prime \prime}(b)}\right|\right)^{i-1}}{(\alpha+2)_{i}}\right. \\
& \left.-\left|f^{\prime \prime}(a)\right| \sum_{i=1}^{\infty}(-1)^{i-1} \frac{\left(\ln \left|\frac{f^{\prime \prime}(a)}{f^{\prime \prime}(b)}\right|\right)^{i-1}}{(\alpha+2)_{i}}\right],
\end{aligned}
$$

for $\left|f^{\prime \prime}(a)\right| \neq\left|f^{\prime \prime}(b)\right|$, and

for $\left|f^{\prime \prime}(a)\right|=\left|f^{\prime \prime}(b)\right|$.

$$
T=\frac{\alpha(b-a)^{2}\left|f^{\prime \prime}(b)\right|}{2(\alpha+1)(\alpha+2)}
$$

Theorem 3.2. Let $f:[a, b] \rightarrow(0, \infty)$ be twice differentiable mapping with $a<b$, $f^{\prime \prime} \in L([a, b])$, satisfying $\left|f^{\prime \prime}(b)\right|>1$. If $\left|f^{\prime \prime}\right|^{q}$ for $q>1$ is $(s, r)$-convex function in the second sense for some fixed $s \in(0,1]$, then the following inequality for fractional integrals holds for $r \geq 0$ and $\alpha>0$

$$
\left|\frac{f(a)+f(b)}{2}-\frac{\Gamma(\alpha+1)}{2(b-a)^{\alpha}}\left[J_{a^{+}}^{\alpha} f(b)+J_{b^{-}}^{\alpha} f(a)\right]\right| \leq T
$$

where

$$
\begin{aligned}
T= & {[c(r)]^{\frac{1}{q}} \frac{(b-a)^{2}}{2(\alpha+1)}\left(\frac{\alpha}{\alpha+2}\right)^{1-\frac{1}{q}}\left[\left|f^{\prime \prime}(a)\right|^{q}+\left|f^{\prime \prime}(b)\right|^{q}\right]^{\frac{1}{q}} } \\
& \times\left[\frac{r^{2}(\alpha+1)}{(s+r)(s+r(\alpha+2))}-\beta\left(\frac{s}{r}+1, \alpha+2\right)\right]^{\frac{1}{q}},
\end{aligned}
$$

for $r>0$,

$$
\begin{aligned}
T= & \frac{(b-a)^{2}}{2(\alpha+1)}\left(\frac{\alpha}{\alpha+2}\right)^{1-\frac{1}{q}}(E(a, b, s, q))^{\frac{1}{q}} \\
& \times\left[\frac{\left|f^{\prime \prime}(a)\right|^{q s}-\left|f^{\prime \prime}(b)\right|^{q s}}{q s \ln \left|f^{\prime \prime}(a)\right|-q s \ln \left|f^{\prime \prime}(b)\right|}-\left|f^{\prime \prime}(b)\right|^{q s} \sum_{i=1}^{\infty} \frac{\left(\ln \left|\frac{f^{\prime \prime}(a)}{f^{\prime \prime}(b)}\right|^{q s}\right)^{i-1}}{(\alpha+2)_{i}}\right.
\end{aligned}
$$




$$
\left.-\left|f^{\prime \prime}(a)\right|^{q s} \sum_{i=1}^{\infty}(-1)^{i-1} \frac{\left(\ln \left|\frac{f^{\prime \prime}(a)}{f^{\prime \prime}(b)}\right|^{q s}\right)^{i-1}}{(\alpha+2)_{i}}\right]^{\frac{1}{q}},
$$

for $r=0$ and $\left|f^{\prime \prime}(a)\right| \neq\left|f^{\prime \prime}(b)\right|$, and

$$
T=\frac{\alpha(b-a)^{2}}{2(\alpha+1)(\alpha+2)}(N(b, s, q))^{\frac{1}{q}},
$$

for $r=0$ and $\left|f^{\prime \prime}(a)\right|=\left|f^{\prime \prime}(b)\right|$, where

$$
E(a, b, s, q)= \begin{cases}\left|f^{\prime \prime}(b)\right|^{q s}, & \text { if }\left|f^{\prime \prime}(a)\right|,\left|f^{\prime \prime}(b)\right| \leq 1, \\ \left|f^{\prime \prime}(b)\right|^{q}, & \text { if }\left|f^{\prime \prime}(a)\right| \leq 1 \leq\left|f^{\prime \prime}(b)\right|, \\ \left|f^{\prime \prime}(a)\right|^{q(1-s)}\left|f^{\prime \prime}(b)\right|^{q s}, & \text { if }\left|f^{\prime \prime}(b)\right| \leq 1 \leq\left|f^{\prime \prime}(a)\right|, \\ \left|f^{\prime \prime}(a)\right|^{q(1-s)}\left|f^{\prime \prime}(b)\right|^{q}, & \text { if }\left|f^{\prime \prime}(a)\right|,\left|f^{\prime \prime}(b)\right| \geq 1,\end{cases}
$$

and

$$
N(b, s, q)= \begin{cases}\left|f^{\prime \prime}(b)\right|^{q s}, & \text { if }\left|f^{\prime \prime}(a)\right|=\left|f^{\prime \prime}(b)\right| \leq 1 \\ \left|f^{\prime \prime}(b)\right|^{q(2-s)}, & \text { if }\left|f^{\prime \prime}(a)\right|=\left|f^{\prime \prime}(b)\right| \geq 1\end{cases}
$$

$c(r)$ is defined as in (3.2) and $(\alpha+2)_{i}=\prod_{j=0}^{i-1}(\alpha+2+j)$.

Proof. Using Lemma 2.2, property of the modulus and power mean inequality, we get

$$
\begin{aligned}
& \left|\frac{f(a)+f(b)}{2}-\frac{\Gamma(\alpha+1)}{2(b-a)^{\alpha}}\left[J_{a^{+}}^{\alpha} f(b)+J_{b^{-}}^{\alpha} f(a)\right]\right| \\
\leq & \frac{(b-a)^{2}}{2(\alpha+1)}\left[\int_{0}^{1}\left[1-(1-t)^{\alpha+1}-t^{\alpha+1}\right] d t\right]^{1-\frac{1}{q}} \\
\times & {\left[\int_{0}^{1}\left[1-(1-t)^{\alpha+1}-t^{\alpha+1}\right]\left|f^{\prime \prime}(t a+(1-t) b)\right|^{q} d t\right]^{\frac{1}{q}} } \\
=\frac{(b-a)^{2}}{2(\alpha+1)}\left(\frac{\alpha}{\alpha+2}\right)^{1-\frac{1}{q}} & {\left[\int_{0}^{1}\left[1-(1-t)^{\alpha+1}-t^{\alpha+1}\right]\left|f^{\prime \prime}(t a+(1-t) b)\right|^{q} d t\right]^{\frac{1}{q}} . }
\end{aligned}
$$

Case 1: $r>0$.

Since $\left|f^{\prime \prime}\right|^{q}$ is $(s, r)$-convex in the second sense, we have

$$
\left|f^{\prime \prime}(t a+(1-t) b)\right|^{q} \leq\left[t^{s}\left|f^{\prime \prime}(a)\right|^{q r}+(1-t)^{s}\left|f^{\prime \prime}(b)\right|^{q r}\right]^{\frac{1}{r}},
$$


the inequality (3.27) can be estimate as

$$
\left|f^{\prime \prime}(t a+(1-t) b)\right|^{q} \leq c(r)\left[t^{\frac{s}{r}}\left|f^{\prime \prime}(a)\right|^{q}+(1-t)^{\frac{s}{r}}\left|f^{\prime \prime}(b)\right|^{q}\right],
$$

where $c(r)$ is defined as in (3.2).

Now, substituting (3.28) into (3.26), we obtain

$$
\begin{aligned}
& \left|\frac{f(a)+f(b)}{2}-\frac{\Gamma(\alpha+1)}{2(b-a)^{\alpha}}\left[J_{a^{+}}^{\alpha} f(b)+J_{b^{-}}^{\alpha} f(a)\right]\right| \\
& \leq[c(r)]^{\frac{1}{q}} \frac{(b-a)^{2}}{2(\alpha+1)}\left(\frac{\alpha}{\alpha+2}\right)^{1-\frac{1}{q}} \\
& \times\left[\left|f^{\prime \prime}(a)\right|^{q} \int_{0}^{1}\left[t^{\frac{s}{r}}-t^{\frac{s}{r}}(1-t)^{\alpha+1}-t^{\frac{s}{r}+\alpha+1}\right] t^{\frac{s}{r}} d t\right. \\
& \left.+\left|f^{\prime \prime}(b)\right|^{q} \int_{0}^{1}\left[(1-t)^{\frac{s}{r}}-(1-t)^{\frac{s}{r}+\alpha+1}-t^{\alpha+1}(1-t)^{\frac{s}{r}}\right] d t\right]^{\frac{1}{q}} \\
& =[c(r)]^{\frac{1}{q}} \frac{(b-a)^{2}}{2(\alpha+1)}\left(\frac{\alpha}{\alpha+2}\right)^{1-\frac{1}{q}}\left[\left|f^{\prime \prime}(a)\right|^{q}+\left|f^{\prime \prime}(b)\right|^{q}\right]^{\frac{1}{q}} \\
& \times\left[\frac{r^{2}(\alpha+1)}{(s+r)(s+r(\alpha+2))}-\beta\left(\frac{s}{r}+1, \alpha+2\right)\right]^{\frac{1}{q}} \cdot
\end{aligned}
$$

Case 2: $r=0$.

Since $\left|f^{\prime \prime}\right|$ is $(s, 0)$-convex, we have

$$
\left|f^{\prime \prime}(t a+(1-t) b)\right|^{q} \leq\left(\left|f^{\prime \prime}(a)\right|^{q}\right)^{t^{s}}\left(\left|f^{\prime \prime}(b)\right|^{q}\right)^{(1-t)^{s}} .
$$

Repeating a similar argument in Theorem 3.1, we obtain

$$
\left|f^{\prime \prime}(t a+(1-t) b)\right|^{q} \leq E(a, b, s, q)\left(\frac{\left|f^{\prime \prime}(a)\right|^{q}}{\left|f^{\prime \prime}(b)\right|^{q}}\right)^{s t}, \quad \text { if }\left|f^{\prime \prime}(a)\right| \neq\left|f^{\prime \prime}(b)\right|,
$$

and

$$
\left|f^{\prime \prime}(t a+(1-t) b)\right|^{q} \leq N(b, s, q) \text { if }\left|f^{\prime \prime}(a)\right|=\left|f^{\prime \prime}(b)\right|,
$$

using (3.32) into (3.26), it yields

$$
\begin{aligned}
& \left|\frac{f(a)+f(b)}{2}-\frac{\Gamma(\alpha+1)}{2(b-a)^{\alpha}}\left[J_{a^{+}}^{\alpha} f(b)+J_{b^{-}}^{\alpha} f(a)\right]\right| \\
\leq & \frac{(b-a)^{2}}{2(\alpha+1)}\left(\frac{\alpha}{\alpha+2}\right)(N(b, s, q))^{\frac{1}{q}} .
\end{aligned}
$$


Now, substituting (3.31) into (3.26) and using the fact that $(1-t)^{n} \leq 2^{1-n}-t^{n}$, we get

$$
\begin{aligned}
& \left|\frac{f(a)+f(b)}{2}-\frac{\Gamma(\alpha+1)}{2(b-a)^{\alpha}}\left[J_{a^{+}}^{\alpha} f(b)+J_{b^{-}}^{\alpha} f(a)\right]\right| \\
\leq & \frac{(b-a)^{2}}{2(\alpha+1)}\left(\frac{\alpha}{\alpha+2}\right)^{1-\frac{1}{q}}(E(a, b, s, q))^{\frac{1}{q}}\left[\int_{0}^{1}\left(\left|\frac{f^{\prime \prime}(a)}{f^{\prime \prime}(b)}\right|^{q s}\right)^{t} d t\right. \\
& \left.-\int_{0}^{1}(1-t)^{\alpha+1}\left(\left|\frac{f^{\prime \prime}(a)}{f^{\prime \prime}(b)}\right|^{q s}\right)^{t} d t-\int_{0}^{1} t^{\alpha+1}\left(\left|\frac{f^{\prime \prime}(a)}{f^{\prime \prime}(b)}\right|^{q s}\right)^{t} d t\right]^{\frac{1}{q}}
\end{aligned}
$$

$$
\begin{aligned}
& =\frac{(b-a)^{2}}{2(\alpha+1)}\left(\frac{\alpha}{\alpha+2}\right)^{1-\frac{1}{q}}(E(a, b, s, q))^{\frac{1}{q}}\left[\frac{\left|f^{\prime \prime}(a)\right|^{q s}-\left|f^{\prime \prime}(b)\right|^{q s}}{q s \ln \left|f^{\prime \prime}(a)\right|-q s \ln \left|f^{\prime \prime}(b)\right|}\right. \\
& \left.\quad-\left|f^{\prime \prime}(b)\right|^{q s} \sum_{i=1}^{\infty} \frac{\left(\ln \left|\frac{f^{\prime \prime}(a)}{f^{\prime \prime}(b)}\right|^{q s}\right)^{i-1}}{(\alpha+2)_{i}}-\left|f^{\prime \prime}(a)\right|^{q s} \sum_{i=1}^{\infty}(-1)^{i-1} \frac{\left(\ln \left|\frac{f^{\prime \prime}(a)}{f^{\prime \prime}(b)}\right|^{q s}\right)^{i-1}}{(\alpha+2)_{i}}\right]^{\frac{1}{q}} .
\end{aligned}
$$

Making as (3.34), (3.33) and (3.29), we get the required inequality in (3.23).

Theorem 3.3. Let $f:[a, b] \rightarrow(0, \infty)$ be twice differentiable mapping with $a<b$, $f^{\prime \prime} \in L([a, b])$. If $\left|f^{\prime \prime}\right|^{q}$ for $q \geq 1$ and $\frac{1}{p}+\frac{1}{q}=1$, is $(s, r)$-convex function in the second sense for some fixed $s \in(0,1]$, then the following inequality for fractional integrals with $\alpha>0$

$$
\left|\frac{f(a)+f(b)}{2}-\frac{\Gamma(\alpha+1)}{2(b-a)^{\alpha}}\left[J_{a^{+}}^{\alpha} f(b)+J_{b^{-}}^{\alpha} f(a)\right]\right| \leq T
$$

where

$$
T=\frac{(b-a)^{2}}{2(\alpha+1)}\left(\frac{p(\alpha+1)-1}{p(\alpha+1)+1}\right)^{\frac{1}{p}}[c(r)]^{\frac{1}{q}}\left(\frac{r}{s+r}\right)^{\frac{1}{q}}\left[\left|f^{\prime \prime}(a)\right|^{q}+\left|f^{\prime \prime}(b)\right|^{q}\right]^{\frac{1}{q}},
$$

for $r>0$,

$$
T=\frac{(b-a)^{2}}{2(\alpha+1)}\left(\frac{p(\alpha+1)-1}{p(\alpha+1)+1}\right)^{\frac{1}{p}}(E(a, b, s, q))^{\frac{1}{q}}\left[\frac{\left|f^{\prime \prime}(a)\right|^{q s}-\left|f^{\prime \prime}(b)\right|^{q s}}{q s \ln \left|f^{\prime \prime}(a)\right|-q s \ln \left|f^{\prime \prime}(b)\right|}\right]^{\frac{1}{q}},
$$

for $r=0$ and $\left|f^{\prime \prime}(a)\right| \neq\left|f^{\prime \prime}(b)\right|$, and

$$
T=\frac{(b-a)^{2}}{2(\alpha+1)}\left(\frac{p(\alpha+1)-1}{p(\alpha+1)+1}\right)^{\frac{1}{p}}(N(b, s, q))^{\frac{1}{q}},
$$

for $r=0$ and $\left|f^{\prime \prime}(a)\right|=\left|f^{\prime \prime}(b)\right|$, holds for $r \geq 0$, where $c(r), E(a, b, s, q)$ and $N(b, s, q)$ are defined as in (3.2), (3.24) and (3.25) respectively. 
Proof. Using Lemma 2.2, property of the modulus and Hölder's inequality, we get

$$
\begin{aligned}
& \left|\frac{f(a)+f(b)}{2}-\frac{\Gamma(\alpha+1)}{2(b-a)^{\alpha}}\left[J_{a^{+}}^{\alpha} f(b)+J_{b^{-}}^{\alpha} f(a)\right]\right| \\
\leq & \frac{(b-a)^{2}}{2(\alpha+1)}\left[\int_{0}^{1}\left[1-(1-t)^{\alpha+1}-t^{\alpha+1}\right]^{p} d t\right]^{\frac{1}{p}}\left[\int_{0}^{1}\left|f^{\prime \prime}(t a+(1-t) b)\right|^{q} d t\right]^{\frac{1}{q}} \\
\leq & \frac{(b-a)^{2}}{2(\alpha+1)}\left[\int_{0}^{1}\left[1-(1-t)^{p(\alpha+1)}-t^{p(\alpha+1)}\right] d t\right]^{\frac{1}{p}} \\
& \times\left[\int_{0}^{1}\left|f^{\prime \prime}(t a+(1-t) b)\right|^{q} d t\right]^{\frac{1}{q}}
\end{aligned}
$$

$$
=\frac{(b-a)^{2}}{2(\alpha+1)}\left(\frac{p(\alpha+1)-1}{p(\alpha+1)+1}\right)^{\frac{1}{p}}\left[\int_{0}^{1}\left|f^{\prime \prime}(t a+(1-t) b)\right|^{q} d t\right]^{\frac{1}{q}}
$$

noting that for any $t \in[0,1], \alpha>0$ and fixed $p \geq 1$

$$
\begin{aligned}
{\left[1-(1-t)^{\alpha+1}-t^{\alpha+1}\right]^{p} } & =\left[\left(1-(1-t)^{\alpha+1}\right)-t^{\alpha+1}\right]^{p} \\
& \leq\left[1-(1-t)^{\alpha+1}\right]^{p}-t^{p(\alpha+1)} \\
& \leq 1-(1-t)^{p(\alpha+1)}-t^{p(\alpha+1)}
\end{aligned}
$$

where we have use the fact that for all $t, n \in[0,1],(1-t)^{n} \leq 2^{1-n}-t^{n}$.

Case 1: $r>0$.

Since $\left|f^{\prime \prime}\right|^{q}$ is $(s, r)$-convex in the second sense, we have

$$
\left|f^{\prime \prime}(t a+(1-t) b)\right|^{q} \leq\left[t^{s}\left|f^{\prime \prime}(a)\right|^{q r}+(1-t)^{s}\left|f^{\prime \prime}(b)\right|^{q r}\right]^{\frac{1}{r}},
$$

and

$$
\left|f^{\prime \prime}(t a+(1-t) b)\right|^{q} \leq c(r)\left[t^{\frac{s}{r}}\left|f^{\prime \prime}(a)\right|^{q}+(1-t)^{\frac{s}{r}}\left|f^{\prime \prime}(b)\right|^{q}\right],
$$

where $c(r)$ is defined as in (3.2).

Using (3.38) into (3.36), we obtain

$$
\begin{aligned}
& \left|\frac{f(a)+f(b)}{2}-\frac{\Gamma(\alpha+1)}{2(b-a)^{\alpha}}\left[J_{a^{+}}^{\alpha} f(b)+J_{b^{-}}^{\alpha} f(a)\right]\right| \\
\leq & \frac{(b-a)^{2}}{2(\alpha+1)}\left(\frac{p(\alpha+1)-1}{p(\alpha+1)+1}\right)^{\frac{1}{p}}[c(r)]^{\frac{1}{q}} \\
& \times\left[\int_{0}^{1} t^{\frac{s}{r}}\left|f^{\prime \prime}(a)\right|^{q} d t+\int_{0}^{1}(1-t)^{\frac{s}{r}}\left|f^{\prime \prime}(b)\right|^{q} d t\right]^{\frac{1}{q}}
\end{aligned}
$$




$$
=\frac{(b-a)^{2}}{2(\alpha+1)}\left(\frac{p(\alpha+1)-1}{p(\alpha+1)+1}\right)^{\frac{1}{p}}[c(r)]^{\frac{1}{q}}\left(\frac{r}{s+r}\right)^{\frac{1}{q}}\left[\left|f^{\prime \prime}(a)\right|^{q}+\left|f^{\prime \prime}(b)\right|^{q}\right]^{\frac{1}{q}} .
$$

Case 2: $r=0$.

Since $\left|f^{\prime \prime}\right|^{q}$ is $(s, 0)$-convex, by a similar argument to Theorem 3.2, we have

$$
\left|f^{\prime \prime}(t a+(1-t) b)\right|^{q} \leq E(a, b, s, q)\left(\frac{\left|f^{\prime \prime}(a)\right|^{q}}{\left|f^{\prime \prime}(b)\right|^{q}}\right)^{s t}, \quad \text { if }\left|f^{\prime \prime}(a)\right| \neq\left|f^{\prime \prime}(b)\right|,
$$

and

$$
\left|f^{\prime \prime}(t a+(1-t) b)\right|^{q} \leq N(b, s, q), \quad \text { if }\left|f^{\prime \prime}(a)\right|=\left|f^{\prime \prime}(b)\right|,
$$

using (3.41) into (3.36), we obtain

$$
\begin{aligned}
& \left|\frac{f(a)+f(b)}{2}-\frac{\Gamma(\alpha+1)}{2(b-a)^{\alpha}}\left[J_{a^{+}}^{\alpha} f(b)+J_{b^{-}}^{\alpha} f(a)\right]\right| \\
\leq & \frac{(b-a)^{2}}{2(\alpha+1)}\left(\frac{p(\alpha+1)-1}{p(\alpha+1)+1}\right)^{\frac{1}{p}}(N(b, s, q))^{\frac{1}{q}} .
\end{aligned}
$$

Now, substituting (3.40) into (3.36), it yields

$$
\begin{aligned}
& \left|\frac{f(a)+f(b)}{2}-\frac{\Gamma(\alpha+1)}{2(b-a)^{\alpha}}\left[J_{a^{+}}^{\alpha} f(b)+J_{b^{-}}^{\alpha} f(a)\right]\right| \\
\leq & \frac{(b-a)^{2}}{2(\alpha+1)}\left(\frac{p(\alpha+1)-1}{p(\alpha+1)+1}\right)^{\frac{1}{p}}(E(a, b, s, q))^{\frac{1}{q}} \\
& \times\left[\frac{\left|f^{\prime \prime}(a)\right|^{q s}-\left|f^{\prime \prime}(b)\right|^{q s}}{q s \ln \left|f^{\prime \prime}(a)\right|-q s \ln \left|f^{\prime \prime}(b)\right|}\right]^{\frac{1}{q}} .
\end{aligned}
$$

From (3.43), (3.42) and (3.39), we get the desired inequality in (3.35). This completes the proof.

Remark 3.4. Theorem 3.3 will be reduced to Theorem 4.1 from [29] also to the Proposition 4.5 from [18], in the case $s=1$.

Theorem 3.4. Suppose that all the assumptions of Theorem 3.3 are satisfied, then the following inequality for fractional integrals with $\alpha>0$, holds

$$
\left|\frac{f(a)+f(b)}{2}-\frac{\Gamma(\alpha+1)}{2(b-a)^{\alpha}}\left[J_{a^{+}}^{\alpha} f(b)+J_{b^{-}}^{\alpha} f(a)\right]\right| \leq T
$$

where

$$
\begin{aligned}
T= & \frac{[c(r)]^{\frac{1}{q}}(b-a)^{2}}{2(\alpha+1)}\left[\left|f^{\prime \prime}(a)\right|^{q}+\left|f^{\prime \prime}(b)\right|^{q}\right]^{\frac{1}{q}} \\
& \times\left[\frac{r^{2} q(\alpha+1)}{(s+r)(s+r q(\alpha+1)+r)}-\beta\left(q(\alpha+1)+1, \frac{s}{r}+1\right)\right]^{\frac{1}{q}},
\end{aligned}
$$


for $r>0$,

$$
\begin{aligned}
T= & \frac{(b-a)^{2}}{2(\alpha+1)}(E(a, b, s, q))^{\frac{1}{q}}\left[\frac{|f(a)|^{q s}-|f(b)|^{q s}}{q s \ln \left|f^{\prime \prime}(a)\right|-q s \ln \left|f^{\prime \prime}(b)\right|}\right. \\
& \left.-\left|f^{\prime \prime}(b)\right|^{q s} \sum_{i=1}^{\infty} \frac{\left(\ln \left|\frac{f^{\prime \prime}(a)}{f^{\prime \prime}(b)}\right|^{q s}\right)^{i-1}}{(q(\alpha+1)+1)_{i}}-\left|f^{\prime \prime}(a)\right|^{q s} \sum_{i=1}^{\infty}(-1)^{i-1} \frac{\left(\ln \left|\frac{f^{\prime \prime}(a)}{f^{\prime \prime}(b)}\right|^{q s}\right)^{i-1}}{(q(\alpha+1)+1)_{i}}\right]^{\frac{1}{q}}
\end{aligned}
$$

for $r=0$ and $\left|f^{\prime \prime}(a)\right| \neq\left|f^{\prime \prime}(b)\right|$, and

$$
T=\frac{(b-a)^{2}}{2(\alpha+1)}(N(b, s, q))^{\frac{1}{q}}\left[\frac{q(\alpha+1)-1}{q(\alpha+1)+1}\right]^{\frac{1}{q}},
$$

for $r=0$ and $\left|f^{\prime \prime}(a)\right|=\left|f^{\prime \prime}(b)\right|$, where $c(r), E(a, b, s, q)$ and $N(b, s, q)$ are defined as in (3.2), (3.24), and (3.25) respectively.

Proof. Using Lemma 2.2, property of the modulus, and Hölder's inequality, we get

$$
\begin{aligned}
& \left|\frac{f(a)+f(b)}{2}-\frac{\Gamma(\alpha+1)}{2(b-a)^{\alpha}}\left[J_{a^{+}}^{\alpha} f(b)+J_{b^{-}}^{\alpha} f(a)\right]\right| \\
\leq & \frac{(b-a)^{2}}{2(\alpha+1)}\left[\int_{0}^{1} d t\right]^{\frac{1}{p}}\left[\int_{0}^{1}\left[1-(1-t)^{\alpha+1}-t^{\alpha+1}\right]^{q}\left|f^{\prime \prime}(t a+(1-t) b)\right|^{q} d t\right]^{\frac{1}{q}} \\
\leq & \frac{(b-a)^{2}}{2(\alpha+1)}\left[\int_{0}^{1}\left[1-(1-t)^{q(\alpha+1)}-t^{q(\alpha+1)}\right]\left|f^{\prime \prime}(t a+(1-t) b)\right|^{q} d t\right]^{\frac{1}{q}} .
\end{aligned}
$$

Case 1: $r>0$.

Since $\left|f^{\prime \prime}\right|^{q}$ is $(s, r)$-convex in the second sense, we have

$$
\left|f^{\prime \prime}(t a+(1-t) b)\right|^{q} \leq\left[t^{s}\left|f^{\prime \prime}(a)\right|^{q r}+(1-t)^{s}\left|f^{\prime \prime}(b)\right|^{q r}\right]^{\frac{1}{r}},
$$

the inequality (3.46) can be reformulated as

$$
\left|f^{\prime \prime}(t a+(1-t) b)\right|^{q} \leq c(r)\left[t^{\frac{s}{r}}\left|f^{\prime \prime}(a)\right|^{q}+(1-t)^{\frac{s}{r}}\left|f^{\prime \prime}(b)\right|^{q}\right],
$$

where $c(r)$ is defined as in (3.2).

Using (3.47) into (3.45), we obtain

$$
\begin{aligned}
& \left|\frac{f(a)+f(b)}{2}-\frac{\Gamma(\alpha+1)}{2(b-a)^{\alpha}}\left[J_{a^{+}}^{\alpha} f(b)+J_{b^{-}}^{\alpha} f(a)\right]\right| \\
\leq & \frac{[c(r)]^{\frac{1}{q}}(b-a)^{2}}{2(\alpha+1)}\left[\left|f^{\prime \prime}(a)\right|^{q} \int_{0}^{1}\left[t^{\frac{s}{r}}-t^{\frac{s}{r}}(1-t)^{q(\alpha+1)}-t^{\frac{s}{r}+q(\alpha+1)}\right] d t\right.
\end{aligned}
$$




$$
\begin{aligned}
& \left.+\left|f^{\prime \prime}(b)\right|^{q} \int_{0}^{1}\left[(1-t)^{\frac{s}{r}}-(1-t)^{\frac{s}{r}+q(\alpha+1)}-t^{q(\alpha+1)}(1-t)^{\frac{s}{r}}\right] d t\right]^{\frac{1}{q}} \\
(3.48)= & \frac{[c(r)]^{\frac{1}{q}}(b-a)^{2}}{2(\alpha+1)} \\
& \times\left[\left|f^{\prime \prime}(a)\right|^{q}\left[\frac{r^{2} q(\alpha+1)}{(s+r)(s+r q(\alpha+1)+r)}-\beta\left(\frac{s}{r}+1, q(\alpha+1)+1\right)\right]\right. \\
& \left.+\left|f^{\prime \prime}(b)\right|^{q}\left[\frac{r^{2} q(\alpha+1)}{(s+r)(s+r q(\alpha+1)+r)}-\beta\left(q(\alpha+1)+1, \frac{s}{r}+1\right)\right]\right]^{\frac{1}{q}} \\
= & \frac{[c(r)]^{\frac{1}{q}}(b-a)^{2}}{2(\alpha+1)}\left[\frac{r^{2} q(\alpha+1)}{(s+r)(s+r q(\alpha+1)+r)}-\beta\left(q(\alpha+1)+1, \frac{s}{r}+1\right)\right]^{\frac{1}{q}} \\
& \times\left[\left|f^{\prime \prime}(a)\right|^{q}+\left|f^{\prime \prime}(b)\right|^{q}\right]^{\frac{1}{q}} .
\end{aligned}
$$

Case 2: $r=0$.

Since $\left|f^{\prime \prime}\right|^{q}$ is $(s, 0)$-convex, by a similar argument to Theorem 3.3, we have

$$
\left|f^{\prime \prime}(t a+(1-t) b)\right|^{q} \leq E(a, b, s, q)\left(\frac{\left|f^{\prime \prime}(a)\right|^{q}}{\left|f^{\prime \prime}(b)\right|^{q}}\right)^{s t}, \quad \text { if }\left|f^{\prime \prime}(a)\right| \neq\left|f^{\prime \prime}(b)\right|,
$$

and

$$
\left|f^{\prime \prime}(t a+(1-t) b)\right|^{q} \leq N(b, s, q), \quad \text { if }\left|f^{\prime \prime}(a)\right|=\left|f^{\prime \prime}(b)\right|,
$$

using (3.50) into (3.45), we obtain

$$
\begin{aligned}
& \left|\frac{f(a)+f(b)}{2}-\frac{\Gamma(\alpha+1)}{2(b-a)^{\alpha}}\left[J_{a^{+}}^{\alpha} f(b)+J_{b^{-}}^{\alpha} f(a)\right]\right| \\
\leq & \frac{(b-a)^{2}}{2(\alpha+1)}(N(b, s, q))^{\frac{1}{q}}\left[\frac{q(\alpha+1)-1}{q(\alpha+1)+1}\right]^{\frac{1}{q}} .
\end{aligned}
$$

Now, substituting (3.49) into (3.45) using Lemma 2.1, we get

$$
\begin{aligned}
& \left|\frac{f(a)+f(b)}{2}-\frac{\Gamma(\alpha+1)}{2(b-a)^{\alpha}}\left[J_{a^{+}}^{\alpha} f(b)+J_{b^{-}}^{\alpha} f(a)\right]\right| \\
\leq & \frac{(b-a)^{2}}{2(\alpha+1)}(E(a, b, s, q))^{\frac{1}{q}}\left[\frac{\left|f^{\prime \prime}(a)\right|^{q s}-\left|f^{\prime \prime}(b)\right|^{q s}}{q s \ln \left|f^{\prime \prime}(a)\right|-q s \ln \left|f^{\prime \prime}(b)\right|}\right. \\
& -\left|f^{\prime \prime}(b)\right|^{q s} \sum_{i=1}^{\infty} \frac{\left(\ln \left|\frac{f^{\prime \prime}(a)}{f^{\prime \prime}(b)}\right|^{q s}\right)^{i-1}}{(q(\alpha+1)+1)_{i}}
\end{aligned}
$$




$$
\left.-\left|f^{\prime \prime}(a)\right|^{q s} \sum_{i=1}^{\infty}(-1)^{i-1} \frac{\left(\ln \left|\frac{f^{\prime \prime}(a)}{f^{\prime \prime}(b)}\right|^{q s}\right)^{i-1}}{(q(\alpha+1)+1)_{i}}\right]^{\frac{1}{q}} .
$$

From (3.48), (3.51) and (3.52), we obtain the desired inequality in (3.44). This completes the proof.

Remark 3.5. Theorem 3.4 will be reduced to Theorem 4.2 from [29], in the case $s=1$.

\section{REFERENCES}

[1] A. O. Akdemir and M. Tunc, On some integral inequalities for s-logarithmically convex functions and their applications, arXiv preprint arXiv:1212.1584.

[2] R.-F. Bai, F. Qi and B.-Y. Xi, Hermite-Hadamard type inequalities for the $m$ - and $(\alpha, m)$ logarithmically convex functions, Filomat 27 (2013), 1-7.

[3] A. Barani, S. Barani and S. Dragomir, Hermite-Hadamard inequalities for functions whose second derivatives absolute values are p-convex, RGMIA Res. Rep. Coll. 14 (2011), Article 73, 8 pp.

[4] A. Barani, S. Barani and S. S. Dragomir, Refinements of Hermite-Hadamard inequalities for functions when a power of the absolute value of the second derivative is p-convex, J. Appl. Math. 2012 (2012), Article 615737, 10 pp.

[5] W. W. Breckner, Stetigkeitsaussagen für eine Klasse Verallgemeinerter Konvexer Funktionen in Topologischen Linearen Räumen, Publ. Inst. Math. (Beograd) (N.S.) 23(37) (1978) , 13-20.

[6] Z. Dahmani, On Minkowski and Hermite-Hadamard integral inequalities via fractional integration, Ann. Funct. Anal. 1 (2010), 51-58.

[7] J. Deng and J. Wang, Fractional Hermite-Hadamard inequalities for $(\alpha, m)$-logarithmically convex functions, J. Inequal. Appl. (2013), Article 364, 11 pp.

[8] S. Dragomir and C. Pearce, Selected Topics on Hermite-Hadamard Inequalities and Applications, RGMIA Monographs, Victoria University, 2000.

[9] S. S. Dragomir and R. P. Agarwal, Two inequalities for differentiable mappings and applications to special means of real numbers and to trapezoidal formula, Appl. Math. Lett. 11 (1998), 91-95.

[10] S. S. Dragomir, J. Pečarić and L. E. Persson, Some inequalities of Hadamard type, Soochow J. Math. 21 (1995), 335-341.

[11] H. Hudzik and L. Maligranda, Some remarks on s-convex functions, Aequationes Math. 48 (1994), 100-111.

[12] İ. İşcan and $\mathrm{S}$. Wu, Hermite-Hadamard type inequalities for harmonically convex functions via fractional integrals, Appl. Math. Comput. 238 (2014), 237-244.

[13] U. N. Katugampola, New approach to a generalized fractional integral, Appl. Math. Comput. 218 (2011), 860-865.

[14] A. A. Kilbas, H. M. Srivastava and J. J. Trujillo, Theory and applications of fractional differential equations, vol. 204 of North-Holland Mathematics Studies, Elsevier Science B.V., Amsterdam, 2006.

[15] U. S. Kirmaci, M. Klaričić Bakula, M. E. Özdemir and J. Pečarić, Hadamard-type inequalities for s-convex functions, Appl. Math. Comput. 193 (2007), 26-35.

[16] W.-H. Li and F. Qi, Some Hermite-Hadamard type inequalities for functions whose $n$-th derivatives are $(\alpha, m)$-convex, Filomat 27 (2013), 1575-1582.

[17] Y. Liao, J. Deng and J. Wang, Riemann-Liouville fractional Hermite-Hadamard inequalities. Part I: for once differentiable geometric-arithmetically s-convex functions, J. Inequal. Appl. (2013), Article 443, 13 pp. 
[18] Z. Lin, J. Wang and W. Wei, Fractional Hermite-Hadamard inequalities through $r$-convex functions via power means, Facta Univ. Ser. Math. Inform. 30 (2015), 129-145.

[19] M. A. Noor, K. I. Noor, M. U. Awan and S. Khan, Fractional Hermite-Hadamard inequalities for some new classes of Godunova-Levin functions, Appl. Math. Inf. Sci. 8 (2014), 2865-2872.

[20] M. A. Noor, F. Qi and M. U. Awan, Some Hermite-Hadamard type inequalities for log-h-convex functions, Analysis (Berlin) 33 (2013), 367-375.

[21] M. E. Özdemir and Ç. Yıldız, New inequalities for Hermite-Hadamard and Simpson type with applications, Tamkang J. Math. 44 (2013), 209-216.

[22] J. Park, Hermite-Hadamard-like type inequalities for s-convex functions and s-Godunova-Levin functions of two kinds, Appl. Math. Sci. 9 (2015), 3431-3447.

[23] C. E. M. Pearce, J. Pečarić and V. Šimić, Stolarsky means and Hadamard's inequality, J. Math. Anal. Appl. 220 (1998), 99-109.

[24] J. E. Pečarić, F. Proschan and Y. L. Tong, Convex functions, partial orderings, and statistical applications, vol. 187 of Mathematics in Science and Engineering, Academic Press, Inc., Boston, MA, 1992.

[25] M. Z. Sarkkaya, On the Hermite-Hadamard-type inequalities for co-ordinated convex function via fractional integrals, Integral Transforms Spec. Funct. 25 (2014), 134-147.

[26] M. Z. Sarikaya and N. Aktan, On the generalization of some integral inequalities and their applications, Math. Comput. Modelling 54 (2011), 2175-2182.

[27] M. Z. Sarikaya, E. Set, H. Yaldiz and N. Başak, Hermite-Hadamard's inequalities for fractional integrals and related fractional inequalities, Mathematical and Computer Modelling 57(9) (2013), 2403-2407.

[28] M. Tunç and H. Kavurmaci-Önalan, On some inequalities for s-logarithmically convex functions in the second sense via fractional integrals, Creat. Math. Inform. 23 (2014), 253-260.

[29] J. Wang, J. Deng and M. Fečkan, Hermite-Hadamard-type inequalities for $r$-convex functions based on the use of Riemann-Liouville fractional integrals, Ukrainian Math. J. 65(2) (2013), 193-211.

[30] J. Wang, X. Li, M. Fečkan and Y. Zhou, Hermite-Hadamard-type inequalities for RiemannLiouville fractional integrals via two kinds of convexity, Appl. Anal. 92 (2013), 2241-2253.

[31] Y. Zhang and J. Wang, On some new Hermite-Hadamard inequalities involving RiemannLiouville fractional integrals, J. Inequal. Appl. (2013), Article 220, 27 pp.

[32] C. Zhu, M. Fečkan and J. Wang, Fractional integral inequalities for differentiable convex mappings and applications to special means and a midpoint formula, J. Appl. Math. Stat. Inform. 8 (2012), 21-28.

\author{
${ }^{1}$ University of 8 May 1945 Guelma, \\ Algeria. \\ E-mail address: khaledv2004@yahoo.fr, tchiheb@yahoo.fr \\ ${ }^{2}$ UNIVERSITY OF BADJI-MOKHTAR, \\ Annaba, Algeria. \\ E-mail address: badrimeftah@yahoo.fr
}

